\title{
Detection of Rice Panicle Blast with Multispectral Radiometer and the Potential of Using Airborne Multispectral Scanners
}

\author{
T. Kobayashi, E. Kanda, K. Kitada, K. Ishiguro, and Y. Torigoe
}

First, second, fourth, and fifth authors: Department of Integrated Research for Agriculture, Tohoku National Agricultural Experiment Station, Morioka 020-0198, Japan; and third author: Nakanihon Air Service Co., Ltd., Tokyo 104-0061, Japan. Accepted for publication 10 November 2000.

\begin{abstract}
Kobayashi, T., Kanda, E., Kitada, K., Ishiguro, K., and Torigoe, Y. 2001. Detection of rice panicle blast with multispectral radiometer and the potential of using airborne multispectral scanners. Phytopathology 91:316-323.

Rice reflectance was measured to determine the spectral regions most sensitive to panicle blast infection. Reflectance increased in the 430- to $530-, 580$ - to $680-$, and $1,480-$ to $2,000-\mathrm{nm}$ regions at the dough stage both in the laboratory and the field as the percentage of diseased

indicators of panicle blast. R470/R570 (reflectance at $470 \mathrm{~nm}$ divided by reflectance at $570 \mathrm{~nm}$ ), R520/R675, and R570/R675 decreased significantly as the incidence of panicle blast increased at the dough stage. At the yellow-ripe stage, R550/R970 and R725/R900 were used to estimate panicle blast severity as measured in terms of the percentage of diseased spikelets. According to the simulation that uses ground-based sensor data, airborne multispectral scanners may be effective in detecting the occurrence of panicle blast using a band combination of 530- to 570- and 650- to 700-nm regions at the dough stage.
\end{abstract} spikelets increased. The wavebands of the greatest sensitivity were in the visible region, located near 485 and $675 \mathrm{~nm}$. After the yellow-ripe growth stage, near-infrared rather than visible reflectance responded to panicle blast infections. Ratios of rice reflectance were evaluated as
Additional keywords: Magnaporthe grisea, measurement condition, remote sensing.
Rice blast disease, caused by Magnaporthe grisea Barr (anamorph Pyricularia grisea Cavara), occurs in rice production areas all over the world and is the most important disease in Japan. Rice blast causes severe damage under cool summer conditions, particularly in northern Japan. The blast pathosystem has two major subsystems, the leaf blast and the panicle blast. During the early growth stages of the host, lesions are mainly formed on leaves, whereas after heading, the pathogen infects the panicles. Panicle blast causes direct yield losses, because grain filling is retarded. The inoculum leading to panicle blast results from the spores formed on the leaf blast lesions. Fungicide application is the most effective method to control rice blast disease. However, it is desirable to minimize the use of agricultural chemicals because of growing environmental concerns as well as cost to the growers. Assessment of disease distribution and severity in nearreal-time before dough stage could provide useful information for making decisions regarding the necessity and appropriate timing of fungicide applications. Currently, disease severity, often rated by field observation, is based on a disease rating standard. The judgment of blast rating may vary with the experience of the individuals (24). The formal investigation by the public pest management staff also has limits related to labor, and it is impossible to accurately estimate the diseased areas and severity over a wide range. Remote sensing techniques may provide an easily available permanent record of disease intensity for large areas without being subjected to raters $(24,29)$.

Aerial photography, ground-based sensor data, and satelliteborne and airborne sensor data have been used to measure disease incidence in many agricultural crops. Bawden (1) first used aerial photography for detecting plant virus diseases of potatoes and

Corresponding author: T. Kobayashi; E-mail address: tkoba@tnaes.affrc.go.jp

Publication no. P-2000-1220-01R

(C) 2001 The American Phytopathological Society tobacco. Thereafter, disease surveys have been conducted on a number of crop species by aerial photography. Examples include cereal rusts (13), potato late blight (4), bacterial blight of beans (18), cotton root rot (41), spot blotch of barley, and powdery mildew of wheat (12). Since the American Landsat 1 was launched on 23 July 1972, several satellites have collected extensive and valuable remote sensing data from agriculture and forestry areas, including data on plant stresses $(22,25,42)$. Sojuzkarta KFA 1000 photographic imagery was used by Torigoe et al. (43) to investigate its feasibility as a means of accurately describing and quantifying soil properties of Andosols and to reveal the relationship between soil properties and the extent of clubroot disease incidence in cabbage. Airborne multispectral scanning is widely studied for surveillance of plant disease, pests, and environmental stresses in agriculture $(19,25)$. The potential of satellite sensor data to detect and monitor plant diseases has led several investigators to examine the spectral responses of crop canopies of diseased plant with ground-based radiometry $(25,30,35)$.

Ground-based sensor data was used to measure the spectral reflectance of corn leaves infected with southern corn leaf blight fungus (34). Corn leaves have greater reflectance after inoculation with Helminthosporium maydis, especially in the 500- to 700- and 1,450- to 1,950-nm-wavelength ranges, than do healthy leaves in the laboratory. Visible leaf reflectance was most sensitive to eight stress agents including pathogens in the 535- to 640- and 685- to 700-nm-wavelength ranges in the laboratory (7). There have also been reports of field experiments using ground-based radiometers to assess the severity of early blight of tomato (21), watermelon disease (2), dollar spot of bentgrass (29), and various leaf and root disease (23). Reflectance at $800 \mathrm{~nm}$ can detect and assess the disease intensity of peanut late leaf spots in the field (28). Nilsson and Johnsson (27) and Nilsson et al. (26) reported significant correlations between near-infrared reflectance (NIR) data (700 to $1,300 \mathrm{~nm}$ ) and infection of anther smut disease in Silene dioica and barley stripe disease. Recently, Raikes and Burpee (32) re- 
ported a significant reduction in reflectance at 760 and $810 \mathrm{~nm}$ as disease severity of Rhizoctonia blight in creeping bentgrass increased.

Though it may be sufficient to analyze only the relative change in reflectance, better information can be obtained by combining data from various spectral ranges. Many formula and vegetation indices, such as spectral ratios, normalized difference indices, and perpendicular vegetative indice, have been developed to reduce multispectral data to a single number for the assessment of physiological characteristics such as leaf area, biomass, nitrogen status, water content, and stress $(8,31,36,37,39)$. In the laboratory, ratios of leaf reflectances that most strongly indicated plant stresses were reflectance at $695 \mathrm{~nm}$ divided by reflectance at 420 or $760 \mathrm{~nm}$ (8). Sharp et al. (35) monitored cereal rust development with a spectral radiometer in the field. The difference of the vegetation index between inoculated and control plants became progressively greater as the rust infection developed. There are no reports related to the use of a ground-based radiometer for the detection and measurement of panicle blast disease.

The objectives of this research were to (i) identify the spectral regions in which rice reflectance was affected by panicle blast infection in the field and in the laboratory at each growth stage of grain development; (ii) select wavebands for ratio computation and determine if the selected ratios could significantly detect and quantify the severities of the disease; and (iii) assess the potential of airborne sensors to detect the occurrence of the disease and to assess differences in disease severity.

\section{MATERIALS AND METHODS}

Spectral reflectance was measured from August to September over a 3-year period, from 1996 to 1998. Several experiments were conducted over this period in field and laboratory conditions. Details are provided in Table 1.

Measurement of rice reflectance at different stages of grain development. Three kilograms of air-dried Andosols was filled into $0.02-\mathrm{m}^{2}$ pots. Two rice seedlings (cv. Akitakomachi), raised in the nursery for 30 days, were transplanted into each pot on 23 May 1996, and the pot was placed in the field to grow. At the yellow-ripe stage, plant height was $108 \mathrm{~cm}$, and the number of tillering was 25 per pot. For reflectance measurements, the potted rice plants were moved to the laboratory. Rice reflectance was measured at the flowering, milky, dough, yellow-ripe, and maturity stage (Fig. 1). After reflectance measurements of whole plants, panicle parts were cut from the neck of the panicle. Spectral reflectances were measured for the whole plant without panicles (vegetative organs) and panicle parts. When measuring the ob-

TABLE 1. Summary of treatments for experiments, results reported in corresponding figures

\begin{tabular}{|c|c|c|c|c|c|c|}
\hline Fig. & Rice $^{\mathrm{a}}$ & Plant part ${ }^{b}$ & Stage $^{c}$ & Planting $^{\mathrm{d}}$ & Location $^{\mathrm{e}}$ & $\mathrm{Cv}^{\mathrm{f}}{ }^{\mathrm{f}}$ \\
\hline 1 & $\mathrm{H}$ & WP,P,VO & $\mathrm{Fl}, \mathrm{Mi}, \mathrm{Do}$ & Pot & $\mathrm{La}$ & $\mathrm{Ak}$ \\
\hline 2 & $\mathrm{H}, \mathrm{D}$ & $\mathrm{P}$ & Do,Ma & Pot & Lab & $\mathrm{Ak}$ \\
\hline 3 & H,D & WP & Do & Pot & Field, Lab & $\mathrm{Sa}$ \\
\hline 4 & $\mathrm{H}, \mathrm{D}$ & WP & $\mathrm{Ye}$ & Field, Pot & Field, Lab & $\mathrm{Ko}, \mathrm{Ak}$ \\
\hline
\end{tabular}

${ }^{a}$ Rice plants were inoculated with Magnaporthe grisea after heading. Healthy plants were used as control (Figs. 2 through 4 ). $\mathrm{H}=$ healthy plants, $\mathrm{D}=$ diseased plants.

${ }^{b}$ After measurement of whole plants (WP), panicle parts were cut from the neck of the panicle and measured for spectral reflectance $(\mathrm{P})$. Similar procedure was applied to vegetative organs (VO, plants without panicles).

${ }^{c}$ Growth stage during grain development when measurements were taken. $\mathrm{Fl}=$ flowering, $\mathrm{Mi}=$ milky, $\mathrm{Do}=$ dough, $\mathrm{Ye}=$ yellow-ripe, and $\mathrm{Ma}=$ maturity.

${ }^{\mathrm{d}}$ Rice plants in the pot or in fields.

${ }^{\mathrm{e}}$ Reflectance measurement was taken in a laboratory using halogen lamps or in the field under sun light.

${ }^{f}$ Three japonica rice cultivars were used. General reflectance responses were similar between Akitakomachi (Ak), Sasanishiki (Sa), and Koshihikari (Ko). jects, whole plants and panicle parts were placed in front of an optically black background and on a black platform, respectively.

Response of rice reflectance to panicle blast infection. The potted plants (cv. Akitakomachi) were inoculated by spraying $20 \mathrm{ml}$ of spore suspension of rice blast isolate Naga 69-150, prepared at $5 \times 10^{5}$ spores per ml. Inoculations were made to plants at the full heading stage on 5 to 7 August 1997. Plants were immediately held in the inoculation box for $10 \mathrm{~h}$ for successful
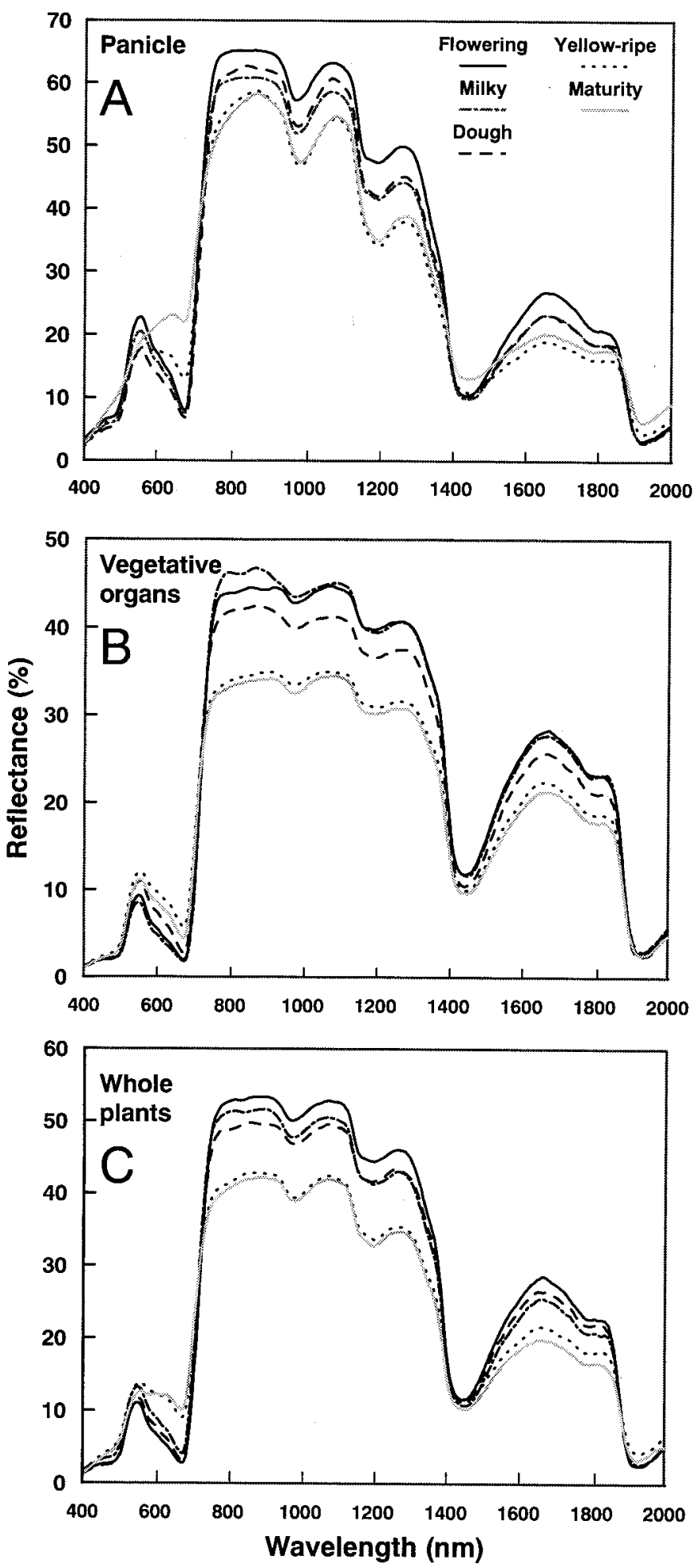

Fig. 1. Reflectance spectra of rice (Oryza sativa L.) plants (cv. Akitakomachi) and plant parts. A, Panicle; B, vegetative organs (whole plants without panicle); and $\mathbf{C}$, whole plants from flowering to maturity growth stages. Measurements were taken under laboratory conditions. 
infection of rice plants by the blast fungus. After inoculating, pots were placed in the field to grow. The potted rice plants were taken to the laboratory for reflectance measurements to identify the spectral region in which rice reflectance was affected by panicle blast infection at each growth stage and in each part of the rice (Fig. 2). Disease incidence was taken manually by assessing the percentage of diseased spikelets.

Seedlings (cv. Sasanishiki) were transplanted to a $3,000-\mathrm{m}^{2}$ field of the Andosols located at the Tohoku National Agricultural Experiment Station on 14 May 1998. Planting density was 20 seedlings per square meter. Basal application of fertilizer was performed at the rate of 40-30-30 kg/ha $\left(\mathrm{N}-\mathrm{P}_{2} \mathrm{O}_{5}-\mathrm{K}_{2} \mathrm{O}\right)$ with an additional application at the rate of $10-10 \mathrm{~kg} / \mathrm{ha}\left(\mathrm{N}-\mathrm{K}_{2} \mathrm{O}\right)$ on 12 July. Panicle blast occurred naturally in the field. Rice plants at the dough stage were uprooted to $0.02-\mathrm{m}^{2}$ pots on 4 September, and reflectance of potted rice plants was measured on the next day both in the field and laboratory (Fig. 3). At the dough stage, plant height was $105 \mathrm{~cm}$, and the number of tillering was 28 per pot.

Rice (cv. Koshihikari) reflectance at the yellow-ripe stage without uprooting was measured in a $3,000-\mathrm{m}^{2}$ field at Yamatsuri, Fukushima Prefecture on 10 September to compare the response in the pot with that in the field (Fig. 4A). Cultural conditions were similar to the field at Tohoku National Agricultural Experiment Station. At the yellow-ripe stage, plant height was $103 \mathrm{~cm}$, and the number of tillering was 24 per plant. Disease incidence was determined by estimating the percentage of diseased spikelets through observation.
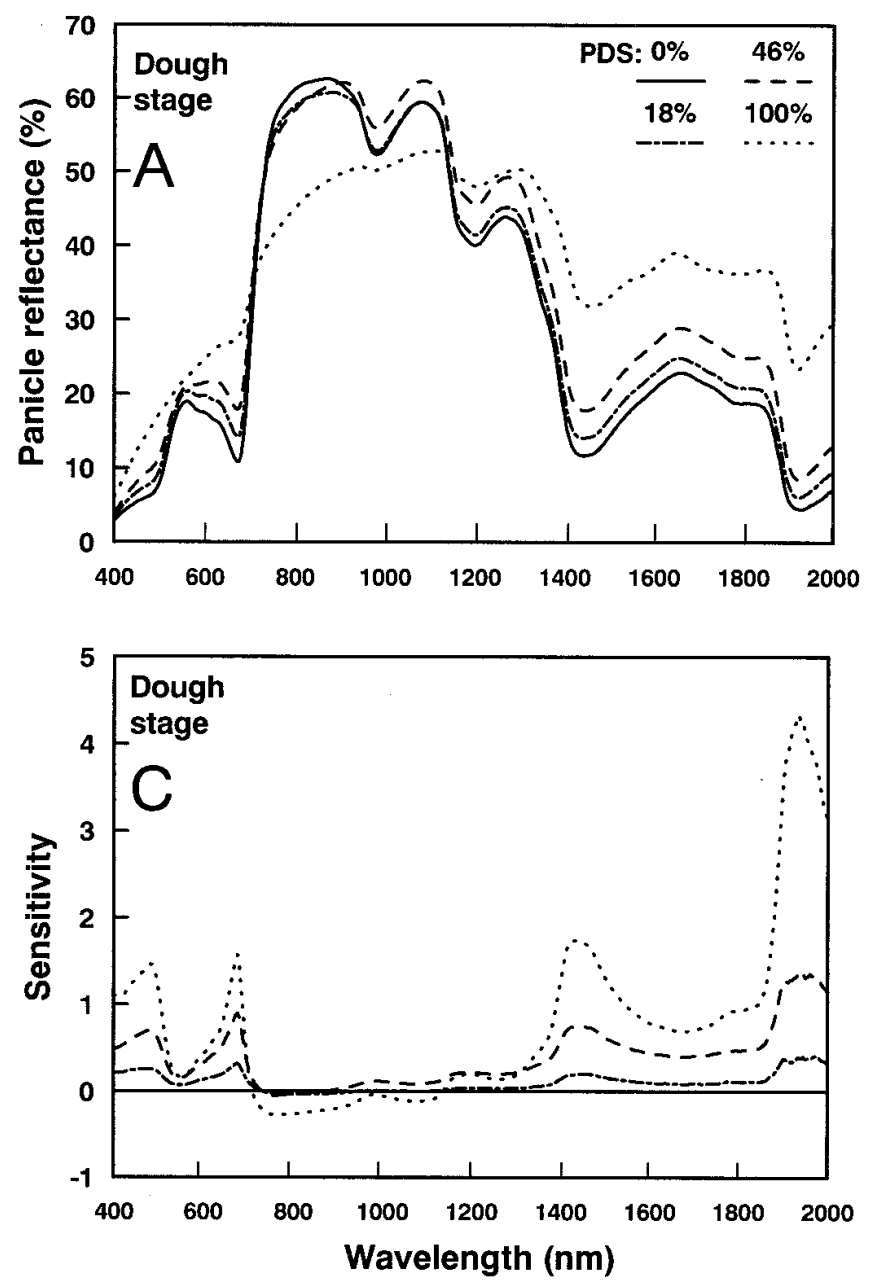

Simulation of airborne multispectral sensors for detecting panicle blast damage based on ground-based sensor data. Table 2 shows the airborne sensors and their bands simulated in this research. The response of these bands to panicle blast infection was simulated with ground-based reflectance. In order to construct the desired airborne bandwidths for analysis, ground-based reflectance measured by spectroradiometer at intervals of $5 \mathrm{~nm}$ were averaged within the bandwidth to form the overall broadband coverages equivalent to the sensor bands (11). For example, band 1 (400 to $460 \mathrm{~nm}$ ) was simulated as averaged reflectance from 400 to $460 \mathrm{~nm}$ at intervals of $5 \mathrm{~nm}$. The reflectance spectrum of uninfected and infected whole plants in the laboratory and field at the dough and yellow-ripe stages was applied for verification. Simulated band ratio was evaluated the potential of detecting blast infection and assessing the disease severity.

Spectroradiometric data. Reflectance was measured with a multispectroradiometer (MSR-7000; Opto Research Corp., Tokyo) that required 3 to $4 \mathrm{~min}$ per scan. Spectral reflectances were measured throughout the 400- to 2,000-nm range because reflected radiances were unsteady and there was a low level of reliability beyond 2,000 $\mathrm{nm}$. The bandwidths of the spectroradiometer channels were $5 \mathrm{~nm}$. The distance between the spectroradiometer and the object was approximately $3 \mathrm{~m}$, allowing radiance measurements of $0.15-\mathrm{m}$ diameter of the object. The spectrometer was mounted on a tripod and held $2 \mathrm{~m}$ above the ground. Reflectances were measured for three pots, each containing a single rice plant. Three to five scans were made for each sample, changing the field
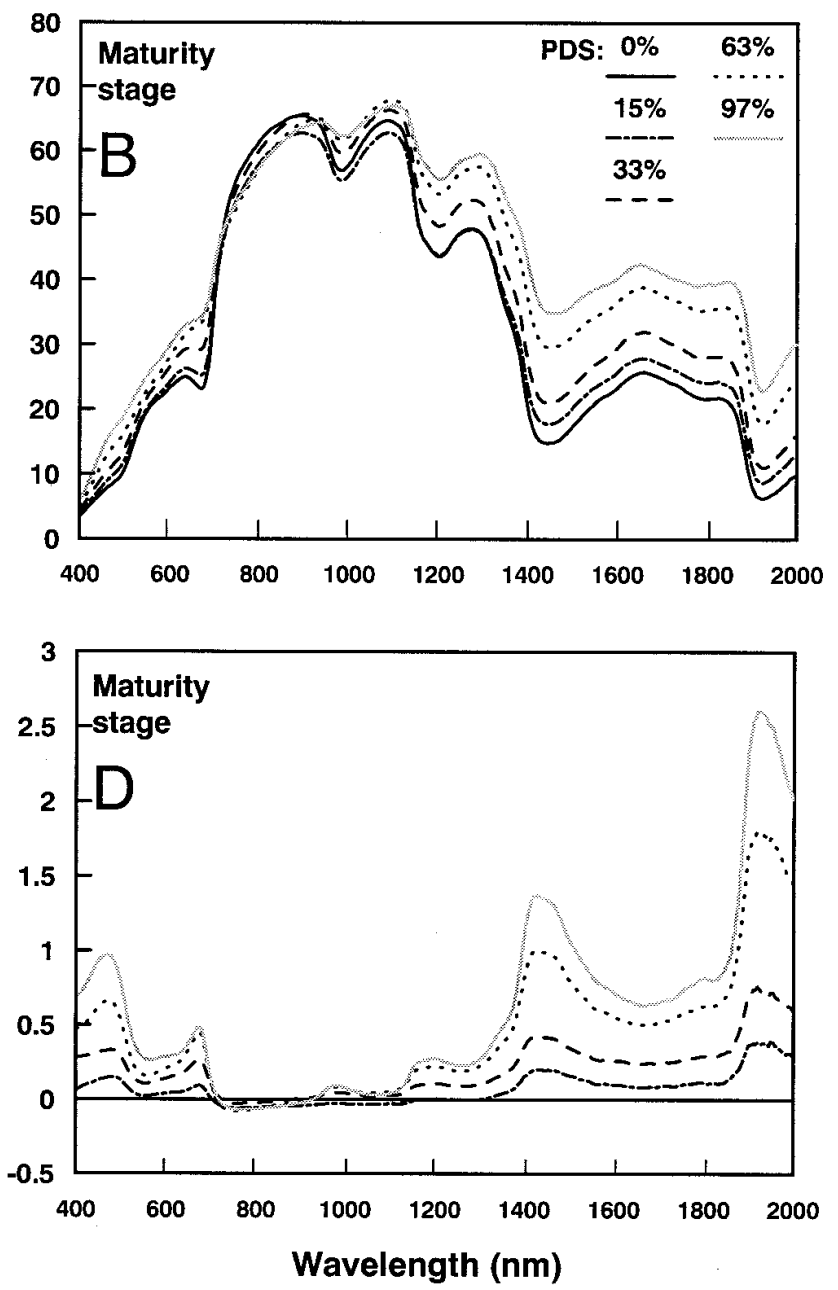

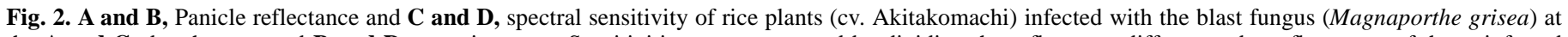

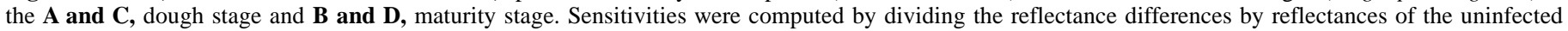
panicles (zero sensitivity). PDS = percentage of diseased spikelets. Measurements were taken under laboratory conditions. 
of view every scan. Reflectance standard $\left(\mathrm{BaSO}_{4}\right.$, Labsphere Inc., North Sutton, NH) measurements were made immediately before and after the spectral measurement of rice plants at the same position in both the field and laboratory experiments. During measurement in the laboratory, the object and the white reference were illuminated by three 500-W halogen lamps. During the field experiments, reflectance was measured only under cloudless conditions from 10 to 12 a.m., when the solar zenith angle was high, resulting in similar irradiance during each experiment.

Data analysis. The radiance reflected from the object was multiplied by 100 and divided by the radiance reflected from the white reference to yield the reflectance percentage. Reflec-
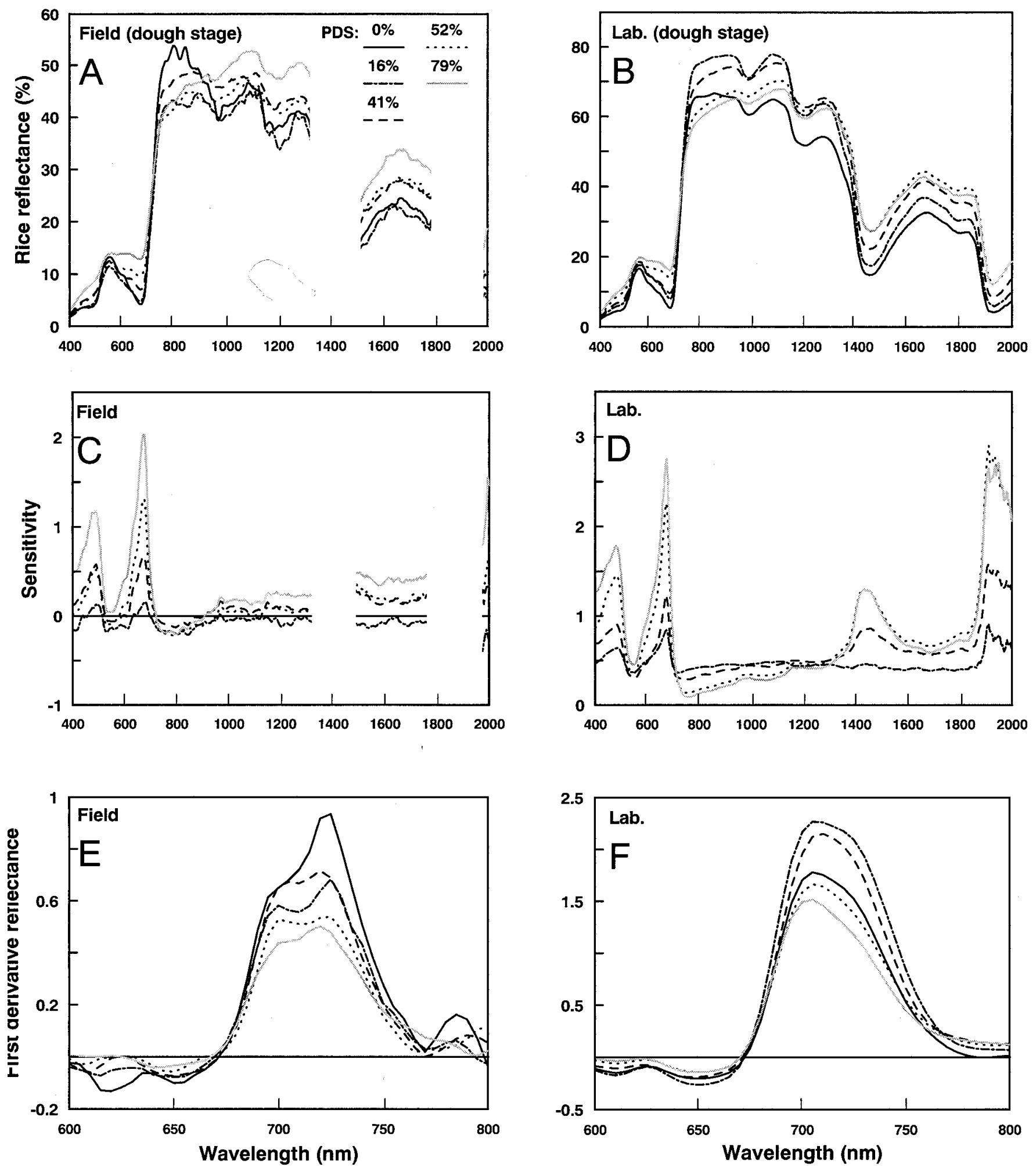

Fig. 3. A and B, Spectral reflectance of whole rice plants (cv. Sasanishiki) infected with blast fungus (Magnaporthe grisea) at the dough stage, C and D, spectral sensitivity to panicle blast infection, and $\mathbf{E}$ and $\mathbf{F}$, the first derivative of the reflectance spectra. $\mathbf{A}, \mathbf{C}$, and $\mathbf{E}$, Reflectance measurements were performed in the laboratory and $\mathbf{B}, \mathbf{D}$, and $\mathbf{F}$, in the field using the same rice plants. The spectral sensitivity was computed dividing the reflectance difference by the reflectance of healthy plants. Naturally infected rice plants in the field were transplanted to pots for reflectance measurement. Note that the red edge occurs between wavelengths of 680 to $750 \mathrm{~nm}$ and is highlighted in the first derivative curve as a peak. PDS = percentage of diseased spikelets. 
tance data were smoothed with a five point moving average to suppress instrumental and environmental noise in the data. The reflectance difference was computed by subtracting the mean reflectance of the uninfected rice from that of the infected rice at each spectroradiometer channel to represent more clearly the reflectance response to the change of disease incidence. The reflectance sensitivity to fungal infection was computed by dividing the appropriate reflectance difference by the mean reflectance of uninfected rice to identify the wavelengths at which reflectance was most strongly affected by fungal infection (6). First, derivative spectra were computed with the first order difference, which is an approximation of the differential in each spectral channel $(5,14)$.

Reflectance difference and sensitivity maxima and minima determined the wavelengths from which numerator and denominator reflectances should be selected for ratio computation (8). The wavelength, based on past research on plant stress and the peaks and bottoms of spectral reflectance, and first derivative curve infected with blast fungus were also used for ratio analysis.

Ratios derived by combining values of percent reflectance in discrete wavelength were calculated to assess their usefulness as indicators of disease severity. Various ratios were tested because different ratios correlate highly with stress as well as other morphological characteristics of vegetative canopies. By combining data from two spectral bands, multispectral radiometric data could be reduced to a single number.
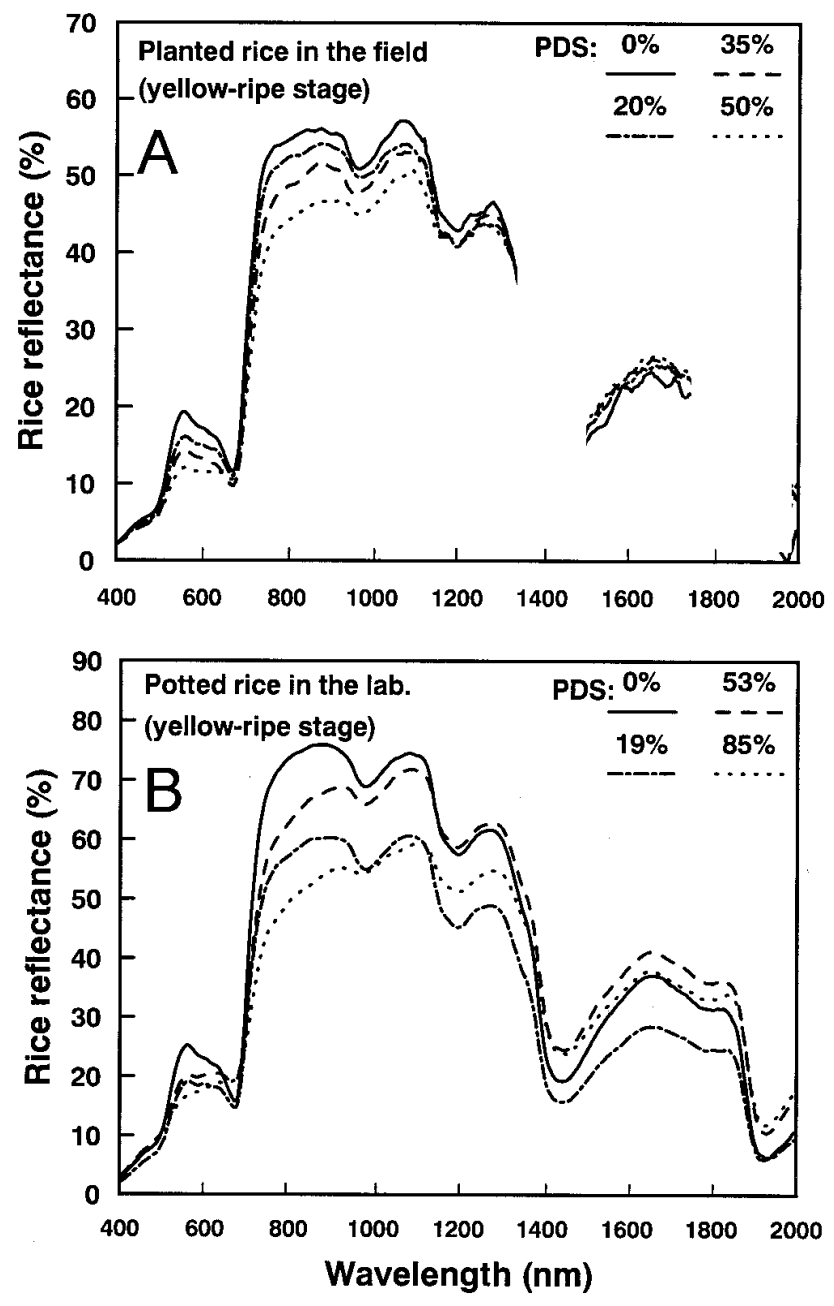

Fig. 4. Spectral reflectance of whole rice plants infected with blast fungus (Magnaporthe grisea) at the yellow-ripe stage. A, Rice plants (cv. Koshihikari) planted in the field. B, Potted rice plants (cv. Sasanishiki) measured in the laboratory. PDS = percentage of diseased spikelets.
Regression analyses were conducted with data of different disease severities in each experiment to determine the extent that ratios were affected by blast fungus infection (32). One regression model was produced for each ratio. Analyses were performed using the Statistical Analysis Software (SAS Institute Inc., Cary, $\mathrm{NC)}$ procedure.

\section{RESULTS}

Reflectance spectrum of the rice plant after the flowering stage in the laboratory. As ripening proceeded from the yellowripe growth stage, panicle reflectance tended to increase in the blue $(400$ to $510 \mathrm{~nm})$ and red $(615$ to $700 \mathrm{~nm})$ regions, where chlorophyll and carotenoid absorb strongly, and decreased in the green (500 to $570 \mathrm{~nm}$ ), NIR and mid-infrared reflectance (MIR: 1,300 to 2,000 nm) ranges (Fig. 1A). Reflectance at $670 \mathrm{~nm}$, the absorption peak of chlorophyll $a$, was approximately $7 \%$ from flowering to dough stage, whereas it was 13 and $22 \%$ at the yellow-ripe and maturity stage, respectively. Reflectance of vegetative organs and whole plants also changed in a similar manner from the yellow-ripe stage in the NIR region (Fig. 1B and C). However, in the 400- to 700-nm spectrum the differences of spectral reflectance during the flowering to the maturity stages for vegetative organs were smaller than that occurring on panicle and whole plants. Whole plant reflectance at $670 \mathrm{~nm}$ at the yellowripe stage was $10 \%$, which is almost an average value between the reflectance of the panicle and the vegetative organs. Because the greatest change in spectral reflectance occurred after the yellowripe stage, analyses of reflectance responses were divided into two periods, from the flowering to the dough stage and from the yellow-ripe to the maturity stage. The reflectance responses of rice cvs. Akitakomachi, Sasanishiki, and Koshihikari were similar at all ripening stages.

Reflectance spectrum of the diseased plants at the dough stage. As the percentage of diseased spikelets increased, panicle reflectance also increased in the visible and MIR regions beyond $1,300 \mathrm{~nm}$ at the dough stage (Fig. 2A). In the NIR region, reflectance differences between uninfected and infected panicles were small with the exception of $100 \%$ of diseased spikelets (Fig. 2A).

The sensitivity of panicle reflectance to the uninfected spikelets was high at the visible wavelengths, with the maxima centered at 480 and $675 \mathrm{~nm}$ (Fig. 2C). The wavebands of greatest sensitivity were in the MIR region, located at 1,435 and 1,935 nm. Sensitivity magnitudes varied with the percentage of diseased spikelets and were greatest at the highest level of disease incidence. Reflectance sensitivity in the NIR region was near zero, regardless of disease incidence.

Reflectance of whole potted plants measured in the laboratory was generally higher than the reflectance measured in the field (Fig. 3A and B). As the percentage of diseased spikelets increased, the reflectance increased in the 430- to 530-nm range,

TABLE 2. Bands and spectral ranges of a simulated airborne sensor

\begin{tabular}{lcc}
\hline Sensor $^{\mathrm{a}}$ & Bands $^{\mathrm{b}}$ & ${\text { Spectral range }(\mathrm{nm})^{\mathrm{c}}}^{\mathrm{d}}$ \\
\hline J-SCAN-AT-5M/II & 1 & $400-460$ \\
& 2 & $490-530$ \\
& 2 & $530-570$ \\
& 3 & $650-700$ \\
& 4 & $950-1,100$ \\
\hline
\end{tabular}

a Airborne Multispectral Scanner (Nakanihon Air Service Co. Ltd., Aichi, Japan). Sensor parameters are IFOV (instantaneous field-of-view) = $2.5 \mathrm{mrad}$, ground resolution $=0.94 \mathrm{~m}$ at an attitude of $300 \mathrm{~m}$, swath width $=$ $480 \mathrm{~m}$ at an attitude of $300 \mathrm{~m}$, total scan angle $=80$ degree, and digitization $=16$ bits (2-bits dummy).

${ }^{b}$ Either band 2 or 2' is available for measurement.

${ }^{\mathrm{c}}$ To simulate an airborne multispectral sensor for detecting panicle blast damage with ground-based reflectance, spectroradiometeric data at 5-nm intervals were averaged within a spectral range. 
580- to 680-nm range, and the MIR regions beyond $1,480 \mathrm{~nm}$ at the dough stage, both in the laboratory and field. Reflectance in the NIR region decreased as the percentage of diseased spikelets increased in the laboratory, whereas the reflectance of whole potted plants was not consistent in the field due to strong winds during measurement. The reflectance of diseased rice was similar to the reflectance of diseased panicles at the dough stage in the visible and MIR ranges (Figs. 2A and 3B).

The wavebands of greatest sensitivity to disease incidence were in the visible region, near 485 and $675 \mathrm{~nm}$, both in the laboratory and in the field (Fig. 3C and D). These wavelengths also showed the greatest sensitivity based on panicle reflectance (Fig. 2C). Sensitivity magnitudes varied with the percentage of diseased spikelets and were greatest at the disease incidence rate of $79 \%$. Reflectance sensitivity to disease incidence in the NIR region was near zero among different disease incidences. The maximum sensitivity of reflectance to disease incidence occurred in the vapor absorption bands near the 1,430- and 1,910- to 1,935-nm regions in the laboratory (Fig. 3D).

The red edge that occurred between wavelengths of 680 to $750 \mathrm{~nm}$ is the point of the maximum slope in vegetation reflectance spectra and is highlighted in the reflectance derivative curve (38). The wavelengths at the peak and the bottom locations in the first derivative curve corresponded, not only regardless of whether they were from the laboratory or the field, but also regardless of disease incidence in the 400- to 680- and 750- to $2,000-\mathrm{nm}$ regions (data not shown). The wavelength of the red edge peak in the laboratory was approximately $705 \mathrm{~nm}$, regardless of panicle blast incidence (Fig. 3F). On the other hand, there were two red edge peaks at approximately 705 and $720 \mathrm{~nm}$ in the field, except in uninfected rice, which was only approximately $725 \mathrm{~nm}$ (Fig. 3E). Single red edge peak of healthy plants in the field showed a shift to wavelengths longer than those in the laboratory.

Reflectance spectrum of diseased plants at the yellow-ripe and the maturity stage. As the percentage of diseased spikelets increased, panicle reflectance tended to increase in the visible and 1,300- to 2,000-nm regions at the maturity stage. (Fig. 2B). However, reflectance differences between uninfected and infected panicles were smaller than the differences observed at the dough stage in the visible region. In the NIR region, there were no apparent reflectance differences between uninfected and infected spikelets at the maturity stage. The maximum sensitivities occurred at 470 and $675 \mathrm{~nm}$ in the visual region (Fig. 2D). The spectral locations of sensitivity maxima were similar not only regardless of the ripening stages, but also regardless of the percentage of diseased spikelets within the 400- to 2,000-nm spectrum. The reflectance of diseased spikelets at the yellow-ripe stage was intermediate between the dough and ripe stage (data not shown).

As disease incidence increased both in the field and in the laboratory at the yellow-ripe stage, the reflectance tended to decrease in the 530 to $655 \mathrm{~nm}$ and NIR regions and increased in the MIR region (Fig. 4A and B). No such difference occurred in the 530- to $655-\mathrm{nm}$ region at the dough stage. These general responses of whole plants were roughly similar between rice planted in the field and potted rice.

Ratios of rice reflectances as indicators of panicle blast and simulation of airborne sensors. The wavelengths selected for ratio computation were based on the above-mentioned results and past research. Regression analysis indicated that the ratios of R570/R675, R470/R570, and R520/R675 at the dough stage and R550/R970 and R725/R900 at the yellow-ripe stage decreased significantly $(P<0.05)$ as the incidence of panicle blast increased (Fig. 5A and B). These ratios were based on the wavelengths of sensitivity maxima and minima (Fig. 3C and D).

To verify the effectiveness of these ratios for airborne sensors, reflectances measured with the multispectral radiometer were averaged in the bandwidth of the sensor (Table 2). Regression analysis indicated that the band ratio of Band 2'/Band 3 at the dough stage decreased significantly $(P<0.05)$ as the incidence of panicle blast increased (Fig. 6). At the yellow-ripe stage, the ratio of Band 2/Band 4 differed significantly $(P<0.05)$ in disease severity.

\section{DISCUSSION}

At the dough stage, increased reflectance of diseased panicle in the blue and red regions, consistently in proportion to the percentage of diseased spikelets, may be attributed to decreased chlorophyll and carotenoid contents in response to the blast infection (Fig. 2A) (20,33). It is thought that the spectral reflectance was strongly influenced by changes in panicle reflectance regardless of measurement conditions after flowering (Figs. 2A and 3A). When the data are presented as reflectance versus wavelength (Fig. 2A), the effects on stress on reflectance are often difficult to evaluate quantitatively $(7,33)$. This is particularly true where the slope of the reflectance curve is large (e.g., the red-infrared transition region). Thus, to represent more clearly the reflectance response to panicle blast damage, reflectance difference and sensitivity were used to identify specific wavelengths in which reflectance was most strongly affected by panicle blast infection $(7,33)$. With plant stress, including exposure to pathogens, reflectance differences are reported to occur in the green and red wavelengths, and major sensitivity peaks are found in the orange $(590$ to $610 \mathrm{~nm})$ and red spectra (610 to $670 \mathrm{~nm})(7)$. In panicle blast infection at the dough stage, however, major reflectance differences and sensitivity peaks occurred in the blue and red regions. It may be that these peaks were estimated based on panicle reflectance rather than leaf reflectance.
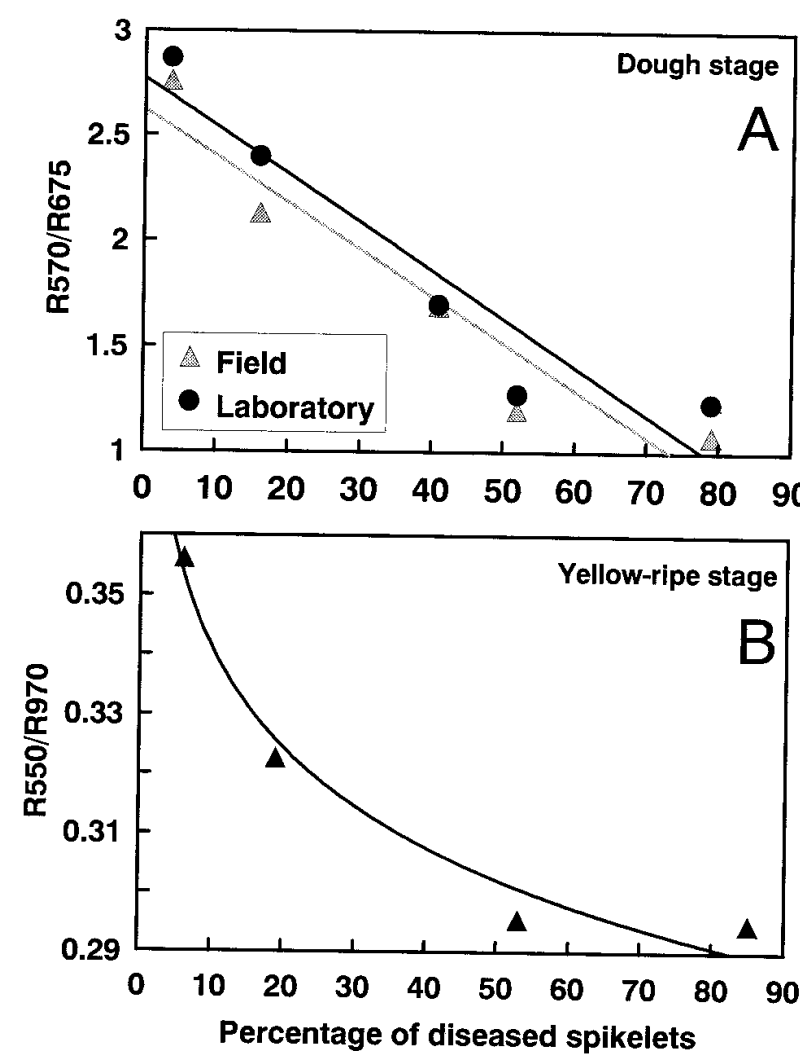

Fig. 5. Relationship between reflectance ratios and percentage of diseased spikelets (PDS) of rice plants infected by Magnaporthe grisea measured at A, the dough stage and B, the yellow-ripe stage. R570/R675 and R550/R970 were calculated from the reflectance data of Figures 3 and $4 \mathrm{~B}$, respectively. Band ratio of each PDS represents means of three to five replicates. A, Field: $y=2.62-0.221 x, r^{2}=0.904$; laboratory: $y=2.77-0.0228 x, r^{2}=0.894$, and B, laboratory: $y=0.397-0.0243(\ln ) x, r^{2}=0.971$. 
At the yellow-ripe stage, the differences between infected and uninfected plants in the blue and red regions were smaller than those occurring in plants at the dough stage. This suggests that the absorption of photosynthetic pigments decreases and the internal structure of the panicle changes during senescence. As disease incidence increases in the MIR region, rice reflectances also rise at all ripening periods. The prominent sensitivity maxima in the water absorption bands centered at 1,435 and 1,935 $\mathrm{nm}$ in the laboratory might indicate the decrease of water content in infected panicles as a result of dehydration. As ripening occurred, it was difficult to distinguish the uninfected and infected spikelets by observation. In the red spectrum, the reflectance difference between infected and uninfected spikelets at the maturity stage was smaller than that at the dough stage, suggesting that chlorophyll content may decrease during ripening. As ripening advanced, the measurement of panicle blast incidence was difficult to detect in the visible spectrum. However, NIR rather than visible reflectance was a more reliable indicator of panicle infection at the maturity stage.

When remote sensing is used for the detection of panicle blast, the sensor data will be applied to the rice reflectance in the field. The reflectance of whole potted plants in the laboratory was compared with that in the field. The wavelengths of the sensitivity maxima were similar regardless of measurement conditions in the visible spectra. Water vapor in the atmosphere strongly absorbs solar radiation in the field. In the visible spectrum, the absorptivity of water vapor is much weaker than in the infrared. There are six major absorption regions within the 700- to 2,000-nm spectrum, roughly in the 700- to 740-, 790- to 840-, 925- to 980-, 1,095 - to $1,165-, 1,320$ - to $1,500-$, and 1,760 - to 1,980 -nm regions. Because absorption regions in the MIR spectrum are especially strong, it was difficult to compare rice reflectance in the laboratory with that in the field. At the dough stage, reflectance response in whole plants uprooted from the field to the pot in the laboratory was similar with that in the field especially in the range of 400 to $1,300 \mathrm{~nm}$. This suggests that data taken in the laboratory can be applicable to field observations. The relationship between rice reflectance planted in the field and reflectance of whole potted plants was examined. The fact that the reflectance within the 400- to 2,000-nm range was similar at the yellow-ripe stage suggests that the results of reflectance in the pot are applicable to rice plants in the field.

The red edge position is the best indicator of plant chlorophyll content $(15,16,40)$. Horler et al. (16) and Boochs et al. (3) defined two components responsible for the position and shape of the red edge peak: chlorophyll content, which causes changes at approxi-

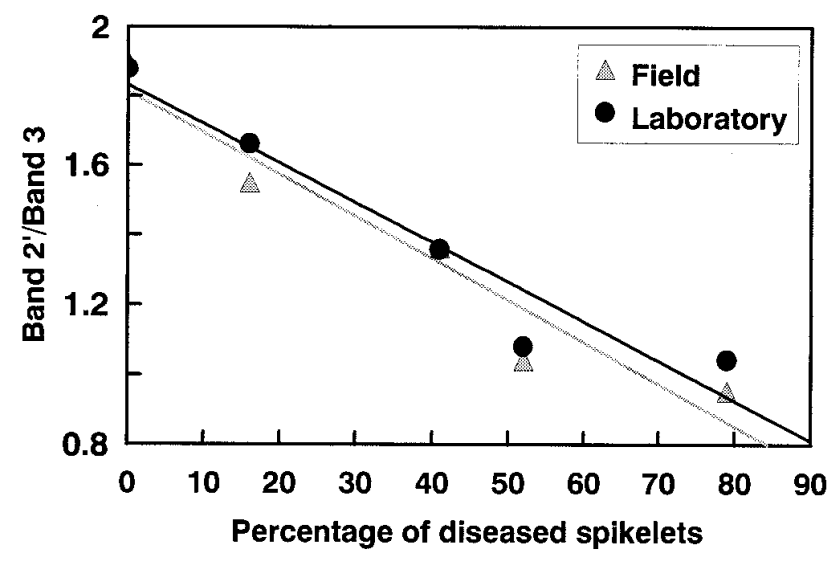

Fig. 6. Relationship between band ratios of an airborne sensor and percentage of diseased spikelets (PDS) of rice plants infected by Magnaporthe grisea measured at the dough stage. Spectral range of bands shown in Table 2. Band ratio of each PDS represents means of three to five replicates. Field: $y=1.81-0.012 x, r^{2}=0.926$; laboratory: $y=1.83-0.0113 x, r^{2}=0.925$. mately $700 \mathrm{~nm}$, and scattering properties, which affect the spectrum at longer wavelengths. These differences in the red edge peak might be explained by the difference of scattering properties between conditions under solar radiation and a halogen lamp or under the absorptivity of water vapor in the 700- to 740-nm region in atmosphere. Furthermore, potted rice reflectance was influenced by the wind speed in the field, especially in the infrared region. This might be explained by differences in the number of tillering between three potted rice plants and rice plants in the field.

Dividing leaf reflectance measured within a stress-sensitive waveband by reflectance measured within a relatively stress-insensitive waveband may largely correct variations in irradiance, leaf orientation, irradiance angles, and shading (8). Reflectance near 695 to $700 \mathrm{~nm}$ divided by reflectance near 670 to $675 \mathrm{~nm}$ indicated precisely the chlorophyll $a$ content of soybean leaves (9). The ratios reported to indicate plant stress are reflectance at $695 \mathrm{~nm}$ divided by reflectance at 420 or $760 \mathrm{~nm}$ (8). In this study, these ratios were not affected by panicle blast infection. It may be that these ratios were evaluated as indicators of plant stress based on leaf reflectance instead of the whole plant reflectance (8). Rice reflectance without the panicle had a small change from the flowering to the maturity stage in the 400- to 2,000-nm wavelength range, and panicle blast symptoms appeared only on the panicle part of rice. R470/R570 and R570/R675, which were based on sensitivity maxima and minima, were also effective in assessing disease severity. Thus, disease incidence may be quantified by measuring the reflectance percentages of these bands. An assessment of diseased areas and disease severities between the flowering and dough stage could be useful for deciding whether or when to apply fungicide for panicle blast control. The maximum sensitivity of reflectance to the infection occurred at 470 and $675 \mathrm{~nm}$ at the dough stage, where carotenoid and chlorophyll $a$ absorb strongly. These sensitivity maxima may be explained by decreases in carotenoid and chlorophyll $a$ contents.

At the yellow-ripe stage, two ratios differed significantly in disease severity (R550/R970 and R725/R900). Absorptivity of photosynthesis pigments was relatively low at approximately $550 \mathrm{~nm}$ at all growth periods. There are several reports regarding the detection of plant water content using NIR reflectance, including wavelengths 970 and 1,195 nm (17) and the linear relationship of total dry mass to the simple difference between the reflectance at 1,100 and $1,200 \mathrm{~nm}$ (36). A similar reduction in NIR reflectance caused by a pathogen infection has been reported in other pathosystems (26-29,32). In this study, it might be difficult to detect panicle blast by the decrease of chlorophyll $a$ and other pigment contents after the yellow-ripe stage, because these contents in the panicle decrease as the plant ripens simultaneously with infection. This suggests that these ratios can be used to quantify disease incidence by changes in panicle water content and biomass. The detection and measurement of disease incidence at the yellow-ripe stage could lead to more reliable estimates of disease loss over large areas. Carter (8) reported that NIR reflectance could be measured in broader bandwidths than is necessary in the visible spectrum to yield a stress-sensitive ratio. In this research, reflectance in any waveband throughout the 900- to 935-nm range could also be divided into reflectance at $725 \mathrm{~nm}$ to produce an infectionsensitive ratio as well as R550/R970 to R1265 at the yellow-ripe stage.

Indices of vegetation studies are computed from digital images with the relatively broad spectral bands of airborne and spaceborne sensors $(42,43)$. However, these bands are generally misplaced spectrally or are too broad to measure vegetation reflectance exclusively within the above stress-sensitive wavebands (8). The combination of stress-insensitive bands with stress-sensitive bands into single broad bands could only decrease the capability of a sensor to detect plant stress (10). To determine if panicle blast disease is actually detectable and assessable by spectral bands of airborne multispectral scanner, we selected the bands for ratio com- 
putation based on the waveband ratios determined by ground-based measurement. The band ratios that were significantly affected by panicle blast infection and offered the greatest potential in remote sensing were determined. This suggests that panicle blast disease is detectable and assessable using the band ratios of airborne sensors. Simple simulation of satellite sensor based on groundbased sensor data was also examined. A few band ratios of satellites distinguished significant differences in disease severity. However, satellites have field of view very different from spectral radiometer. Bidirectional reflectance distribution function models such as SAIL (scattering by arbitrary inclined leaves) that describe light interaction in vegetation canopies may assist in predicting the spectral reflectance of canopy $(11,44)$. In speculating about the extension of techniques described in this paper to an airborne system to monitor panicle blast incidence, it must be taken into consideration that the sensor data acquired from airplanes can be severely affected by atmospheric conditions. Photography or digital imaging within spectrally narrow ranges may provide an improved capability to detect panicle blast. There is an apparent feasibility in the use of remotely collected radiometric reflectance data to estimate disease incidence over large production areas, and this information could also be used to decide the necessity of fungicide application or to estimate potential yield losses due to the disease.

\section{ACKNOWLEDGMENTS}

We thank T. Nakajima, H. Tohjoh, and K. Nemoto at Fukushima Prefectural Agricultural Experiment Station for valuable discussion, and Y. Ohmori, K. Kudoh, and G. Fujimura at Tohoku National Agricultural Experiment Station for their technical assistance.

\section{LITERATURE CITED}

1. Bawden, F. C. 1933. Infra-red photography and plant virus diseases. Nature 132:168.

2. Blazquez, C. H., and Edwards, G. J. 1986. Spectral reflectance of healthy and diseased watermelon leaves. Ann. Appl. Biol. 108:243-249.

3. Boochs, F., Kupfer, G., Dockter, K., and Kübauch, W. 1990. Shapes of the red edge as vitality indicator for plants. Int. J. Remote Sens. 11: 1741-1753.

4. Brenchley, G. H. 1968. Aerial photography for the study of plant diseases. Annu. Rev. Phytopathol. 6:1-22.

5. Bulter, W. L., and Hopkins, D. W. 1970. Higher derivative analysis of complex absorption spectra. Photochem. Photobiol. 12:439-450.

6. Carter, G. A. 1991. Primary and secondary effects of water content on the spectral reflectance of leaves. Am. J. Bot. 78:916-924.

7. Carter, G. A. 1993. Response of leaf spectral reflectance to plant stress. Am. J. Bot. 80:239-243.

8. Carter, G. A. 1994. Ratios of leaf reflectances in narrow wavebands as indicators of plant stress. Int. J. Remote Sens. 15:697-703.

9. Chappelle, E. W., Kim, M. S., and McMurtrey, J. E. 1992. Ratio analysis of reflectance spectra (RARS): An algorithm for the remote estimation of the concentrations of chlorophyll a, chlorophyll b, and carotenoids in soybean leaves. Remote Sens. Environ. 39:239-247.

10. Cibula, W. G., and Carter, G. A. 1992. Identification of a far-red reflectance response to ectomycorrhizae in slash pine. Int. J. Remote Sens. 13:925-932.

11. Cibula, W. G., Zetka, E. F., and Rickman, D. L. 1992. Response of thematic mapper bands to plant stress. Int. J. Remote Sens. 13:1869-1880.

12. Clark, R. V., Galway, D. A., and Paliwal, Y. C. 1981. Aerial infrared photography for disease detection in field plots of barley, oats and wheat. (Abstr.) Phytopathology 71(suppl.):S867.

13. Clowell, R. N. 1956. Determining the prevalence of certain cereal diseases by means of aerial photography. Hilgardia 26:223-286.

14. Danson, F. M., Steven, M. D., Malthus, T. J., and Clark, J. A. 1992. High-spectral resolution data for determining leaf water content. Int. J. Remote Sens. 13:461-470.

15. Filella, I., and Peñuelas, J. 1994. The red edge position and shape as indicators of plant chlorophyll content, biomass and hydric status. Int. J. Remote Sens. 15:1459-1470.

16. Horler, D. N. H., Dockray, M., and Baeber, J. 1983. The red edge of plant leaf reflectance. Int. J. Remote Sens. 4:273-278.

17. Hunt, E. R., Jr., and Rock, B. N. 1989. Detection of change in leaf water content using near- and middle-infrared reflectances. Remote Sens.
Environ. 30:43-54.

18. Jackson, H. R., and Wallen, V. R. 1975. Microdensitometer measurements of sequential aerial photographs of field beans infected with bacterial blight. Phytopathology 65:961-968.

19. Jackson, R. D. 1986. Remote sensing of biotic and abiotic plant stress. Annu. Rev. Phytopathol. 24:265-287.

20. Koga, H. 1995. An electron microscopic study of the infection of spikelets of rice by Pyricularia oryzae. J. Phytopathol. 143:439-445.

21. Lathrop, L. D., and Pennypacker, S. 1980. Spectral classification of tomato disease severity levels. Photogram. Eng. Remote Sens. 46:1433-1438.

22. Nagarajan, S., Seibold, G., Kranz, J., Saari, E. E., and Joshi, L. M. 1984. Monitoring wheat rust epidemics with the Landsat-2 satellite. Phytopathology 74:585-587.

23. Nilsson, H. E. 1991. Hand-held radiometry and IR thermography of plant diseases in field plot experiments. Int. J. Remote Sens. 12:545-557.

24. Nilsson, H. E. 1995. Remote sensing and image analysis in plant pathology. Annu. Rev. Phytopathol. 15:489-527.

25. Nilsson, H. E. 1995. Remote sensing and image analysis in plant pathology. Can. J. Plant Pathol. 17:154-166.

26. Nilsson, M., Elmqvist, T., and Carlsson, U. 1994. Use of near-infrared reflectance spectrometry and multivariate data analysis to detect anther smut disease (Microbotryum violaceum) in Silene dioica. Phytopathology 84:764-770.

27. Nilsson, H. E., and Johnsson, L. 1996. Hand-held radiometry of barley infected by barley stripe in a field experiment. J. Plant Dis. Prot. 103:517-526.

28. Nutter, F. W., Jr. 1989. Detection and measurement of plant disease gradients in peanut with a multispectral radiometer. Phytopathology 79:958-963.

29. Nutter, F. W., Jr., Gleason, M. L., Jenco, J. H., and Christians, N. C. 1993. Assessing the accuracy, intra-rater repeatability, and inter-rater reliability of disease assessment systems. Phytopathology 83:806-812.

30. Pennypacker, S. P., Scharen, A. L., Sharp, E. L., and Sands, D. C. 1982. Spectral classification of wheat infected with barley yellow dwarf and stripe rust. (Abstr.) Phytopathology 72(suppl.):S1006.

31. Peñuelas, J., Filella, I., Biel, C., Serrano, L., and Savé, R. 1993. The reflectance at the $950-970 \mathrm{~nm}$ regions as an indicator of plant status. Int. J. Remote Sens. 14:1887-1905.

32. Raikes, C., and Burpee, L. L. 1998. Use of multispectral radiometry for assessment of Rhizoctonia blight in creeping bentgrass. Phytopathology 88:446-449.

33. Riedell, W. E., and Blackmer, T. M. 1999. Leaf reflectance spectra of cereal aphid-damaged wheat. Crop Sci. 39:1835-1840.

34. Safir, G. R., Svits, G. H., and Ellingboe, A. H. 1972. Spectral reflectance and transmittance of corn leaves infected with Helminthosporium maydis. Phytopathology 62:1210-1213.

35. Sharp, E. L., Perry, C. R., Scharen, A. L., Boatwright, G. O., Sands, D. C., Lautenschlager, L. E., Yahyaoui, C. M., and Ravet, F. W. 1985. Monitoring cereal rust development with a spectral radiometer. Phytopathology 75:936-939.

36. Shibayama, M., and Akiyama, T. 1989. Seasonal visible, near-infrared and mid-infrared spectra of rice canopies in relation to LAI and aboveground dry phytomass. Remote Sens. Environ. 27:119-127.

37. Shibayama, M., Wiegand, C. L., Akiyama, T., and Yamagata, T. 1988. Radiometric predictions for agronomic variables of rice canopies using a visible to mid-infrared spectroradiometer. Int. Arch. Photogram. Remote Sens. 7:508-517.

38. Steven, M. D., Malthus, T. H., Demetriades-Shah, T. H., Danson, F. M., and Clark, J. A. 1990. High spectral resolution indices for crop stress. Pages 209-228 in: Application of Remote Sensing in Agriculture. M. D. Steven and J. A. Clark, eds. Butterworths, London.

39. Takebe, M., Yoneyama, T., Inada, K., and Murakami, T. 1990. Spectral reflectance ratio of rice canopy for estimating crop nitrogen status. Plant Soil 122:295-297.

40. Thomas, J. R., and Gausman, H. W. 1977. Leaf reflectance versus leaf chlorophyll and carotenoid concentration for eight crops. Agron. J. 69:799-802.

41. Toler, R. W., Smith, B. D., and Harlan, J. C. 1981. Use of aerial color infrared to evaluate crop disease. Plant Dis. 65:24-31.

42. Torigoe, Y., Amano, T., Ogawa, K., and Fukuhara, M. 1992. Discrimination of cabbage fields for detecting clubroot disease using Landsat thematic mapper data. Jpn. J. Crop Sci. 64:527-535.

43. Torigoe, Y., Inoue, T., Tmano, T., Ogawa, K., and Fukuhara, M. 1993. Mapping soil properties of Andosols and assessment of clubroot disease incidence in cabbage using Sojuzkarta KFA 1000 photographic imagery. Jpn. J. Crop Sci. 62:585-594.

44. Verhoef, W. 1984. Light scattering by leaf layers with application to canopy reflectance modeling: The SAIL model. Remote Sens. Environ. $16: 125-141$. 